\title{
Über die Einwirkung von Phosphorpenta- sulfid auf Harnstoff und Thioharnstoff
}

\author{
von \\ Franz v. Hemmelmayr.
}

Aus dem chemischen Laboratorium der Landes-Oberrealschule in Graz.

(Vorgelegt in der Sitzung am 6. April 1905.)

Vor mehr als 15 Jahren wurde im Laboratorium des Herrn Prof. Dr. Maly in Prag die Einwirkung von Phosphorpentasulfid auf Harnstoff $\mathrm{zu}$ dem $Z_{w}$ ecke studiert, um auf diese Weise eventuell Thioharnstoff darzustellen. Dies gelang nicht, dagegen erhielt Herr Erhardt bei dieser Gelegenheit einen schön kristallisierenden schwefel- und phosphorhältigen Körper, der später von Herrn Karl v. Kutschig genauer untersucht wurde. ${ }^{1} \mathrm{Kutschig}$ beschreibt die Darstellung der Verbindung, teilt die Messung ihrer Kristallformen, einige Analysen, die Einwirkung von Bleioxyd und von Salzsäure sowie einige Versuche mit, die die Stellung der Stickstoffund Schwefelatome aufklären sollen; auch wird die Darstellung und Analyse eines Silbersalzes besprochen. Auf Grund seiner Versuche stellt Kutschig die empirische Formel $\mathrm{C}_{2} \mathrm{H}_{8} \mathrm{~N}_{3} \mathrm{PS}_{2} \mathrm{O}_{2}$ und die allerdings mit Vorbehalt gegebene Strukturformel

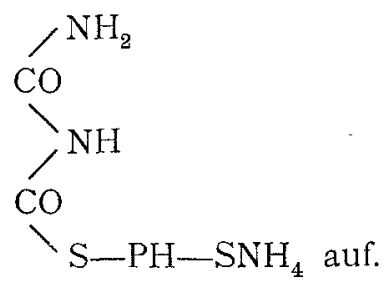

1 Monatshefte für Chemie, 9, 406. 
Da mir letztere Formel stets sehr unwahrscheinlich erschien, habe ich, durch andere Versuche an jene Verbindung erinnert, das Studium der Reaktion zwischen Harnstoff und Phosphorpentasulfid wieder aufgenommen.

Ich habe dabei zunächst die Beobachtung gemacht, daß der Verlauf der Reaktion sehr von den Versuchsbedingungen abhängt, indem nur unter Einhaltung bestimmter Bedingungen stets das gleiche Produkt entsteht. Trotzdem ich mit denselben relativen Mengen von Harnstoffund Phosphorsulfid wie Kuts chig arbeitete, konnte ich aber nie beobachten, daß der neue Körper leichter löslich sei als das gleichzeitig gebildete Ammoniumphosphat, bei mir war es stets umgekehrt; infolgedessen schied sich auch die neue Verbindung beim Eindampfen der Lösung des Reaktionsproduktes stets zuerst aus. Da auch meine Analysen zum Teile sogar erheblich von denen Kutschig's abwichen, könnte es zweifelhaft erscheinen, $a b$ ich denselben Körper wie er in Händen hatte. Die kristallographische Messung stellte aber unzweifelhaft die Identität beider fest.

Auf Grund meiner zahlreichen an Material von verschiedenen Darstellungen angestellten Analysen kam ich zur empirischen Formel $\mathrm{C}_{2} \mathrm{H}_{7} \mathrm{~N}_{4} \mathrm{PS}_{2} \mathrm{O}_{2}$ für die Verbindung. Kutschig's Formel enthält ein Stickstoffatom weniger als meine. Da der Körper ein Ammoniumsalz ist, so war Kutschig's Verbindung voraussichtlich durch freie Säure verunreinigt, worauf auch die Löslichkeitsverhältnisse hindeuten. Zur Kristallmessung können ja zufällig Kristalle des reinen Ammoniumsalzes verwendet worden sein.

$\mathrm{Da}$ das Reaktionsprodukt saure Eigenschaften besitzt, war die Darstellung von Salzen wünschenswert, einerseits um die Basizität, andrerseits auch um das Molekulargewicht festzustellen. Kutschig wählte hiezu das Silbersalz, bekam aber keine befriedigenden Resultate. Ich konnte zeigen, daß sein primäres Silbersalz für meine Formel gute Übereinstimmung liefert.

Wegen der Lichtempfindlichkeit der Silbersalze habe ich die Darstellung eines Bariumsalzes vorgezogen.

Es ist mir gelungen, ein völlig einheitliches Baryumsalz darzustellen, aus dessen Analyse hervorgeht, daß die Säure 
dreibasisch ist und jedenfalls das einfache Molekulargewicht $\mathrm{C}_{2} \mathrm{H}_{7} \mathrm{~N}_{4} \mathrm{PS}_{2} \mathrm{O}_{2}$ besitzt; auch ergab sich, daß bei der Bildung des tertiären Salzes ein Molekül Wasser aufgenommen wird.

Ein tertiäres Ammoniumsalz ist zwar erhältlich, es zersetzt sich aber schon beim Liegen an der Luft unter Abgabe von Ammoniak und Bildung des primären Salzes.

Mit Hilfe des Baryumsalzes gelang auch die Darstellung der freien Säure, indem ersteres mit den berechneten Mengen von Schwefelsäure zersetzt wurde. Die freie Säure ist sehr leicht löslich und konnte nur in kristallwasserhältigem Zustand analysiert werden. Durch Überführen der freien Säure in primäres Ammoniumsalz ließ sich die Reinheit, beziehungsweise Einheitlichkeit des ursprünglichen Reaktionsproduktes nachweisen sowie auch zeigen, daß bei der Überführung in Baryumsalz und Zersetzung desselben mit Schwefelsäure eine weitergehende Spaltung nicht stattgefunden hatte.

Schon Kutschig hatte die Spaltung der Verbindung in Schwefelwasserstoff, Biuret und Phosphorsäure durch verdünnte Salzsäure beobachtet, behauptet aber, daß bei Luftabschlup hiebei phosphorige Säure entsteht. Ich habe nun nachgewiesen, daß dies nicht der Fall ist, sondern daß auch unter diesen Umständen lediglich Phosphorsäure gebildet wird.

Die glatte Zersetzung in Schwefelwasserstoff, Biuret und Phosphorsäure durch verdünnte Säuren veranlassen mich, der Verbindung, die überdies ein Ammoniumsalz ist, den Namen thiobiuretphosphorsaures Ammonium und der zu Grunde liegenden Säure den Namen Thiobiuretphosphorsäure beizulegen. Was die Konstitution der Verbindung anbelangt, so muß vor allem berücksichtigt werden, daß der Biuretrest schon fertig gebildet vorliegen, ebenso auch der Phosphor in einer Bindungsform vorhanden sein muß, die den leichten Zerfall in Biuret und Phosphorsäure erklärlich machen.

Dies wäre der Fall, wenn es sich um einen Phosphorsäureester handeln würde, doch spricht dagegen der niedrige Sauerstoffgehalt. Es kann also der Phosphor nur zwei NHGruppen verbinden, indem bei der Reaktion zwischen zwei $\mathrm{NH}_{2}$-Gruppen und einem Schwefelatom des Pentasulfides Schwefelwasserstoff ausgetreten ist. Es kommt dadurch eine 
analoge Struktur zu stande, wie sie beispielsweise in den Ureïden vorliegt.

Dafür, daß die Thiobiuretphosphorsäure keine Amidogruppe enthält, spricht auch ihr stark saurer Charakter, der doch sonst eine wesentliche Schwächung erfahren müßte, während er so durch die Imidgruppe verstärkt wird.

Da die substituierten Dithiobiurete, wie ich mich am Methyldithiobiuret, das mir Herr Prof. O. Hecht in liebenswürdiger Weise zur Verfügung stellte, überzeugt habe, beim Kochen mit Salzsäure keinen Schwefelwasserstoff entwickeln, so ist wohl ausgeschlossen, daß die Thiobiuretphosphorsäure sich vom Dithiobiuret ableitet. Es bleibt dann nur die Möglichkeit, daß die Schwefelatome an Phosphor gebunden sind, entsprechend folgender Formel:

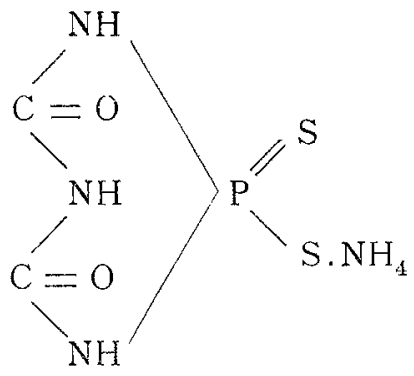

Man könnte zwar dagegen einwenden, daß das thiobiuretphosphorsaure Ammonium durch kalte Salzsäure (auch konzentrierte) nicht unter Schwefelwasserstoffentwicklung zersetzt wird, während die freien Thiophosphorsäuren schon durch Wasser sehr leicht in die entsprechenden Sauerstoffverbindungen übergehen, doch ist anderseits wieder zu bedenken, daß auch der Biuretkomplex einen wesentlichen Einfluß auf die Eigenschaften der Verbindung haben wird und damit auch die Schwefelatome eine festere Bindung im Molekül erhalten können.

Für diese Auffassung könnte auch angeführt werden, daß bei der Abspaltung des Biurets durch Kochen mit verdünnten Säuren auch der Schwefel als Schwefelwasserstoff weggeht, wenn auch nicht festgestellt, werden kann, ob die beiden 
Prozesse nacheinander oder gleichzeitig stattfinden. Der Fall, daß ein Schwefelatom im Biuretkomplex das andere an Phosphor gebunden ist, ist so wenig wahrscheinlich, daß eine nähere Diskussion hierüber überflüssig ist.

Da bei der Bildung der tertiären Salze ein Molekül Wasser aufgenommen wird, leiten sich diese von einer Säure der Zusammensetzung

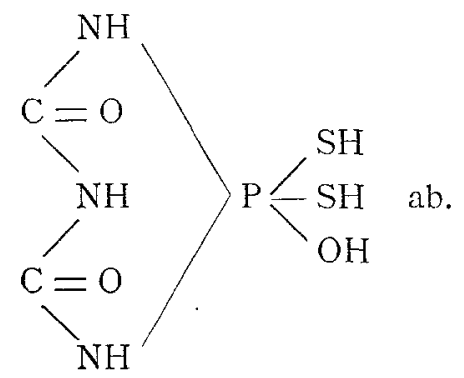

Ich habe versucht, auch durch Einwirkung von Phosphorpentasulfid auf Thioharnstoff die Frage nach der Stellung der Schwefelatome zu entscheiden, doch wirken die beiden Stoffe bei den Versuchsbedingungen scheinbar nicht aufeinander ein, oder sie bilden doch nur ein lockeres, schon durch Wasser wieder spaltbares Reaktionsprodukt.

\section{Experimenteller Teil.}

$100 \mathrm{~g}$ Harnstoff wurden mit $200 \mathrm{~g}$ fein gepulvertem Phosphorpentasulfid innig verrieben und die Mischung in einem Glaskolben auf dem Wasserbade eine halbe Stunde erwärmt. Die blasige Masse wurde mit kaltem Wasser übergossen, wobei sich stürmisch Schwefelwasserstoff entwickelte; als die Gasentwicklung nur noch ganz schwach vor sich ging, wurde filtriert und das Filtrat eingedampft. Die zuerst ausgefallenen Kristalle (I) enthielten noch geringe Mengen Ammoniumphosphat, von dem durch Umkristallisieren aus heißem Wasser getrennt wurde. Es hatte sich also nicht, wie Kutschig angibt, zuerst Ammoniumphosphat ausgeschieden. Das Filtrat von I lieferte bei weiterem Eindampfen einen kristallinischen Rückstand (die überwiegende Menge des Reaktionsproduktes 
vorstellend), der viel Ammoniumphosphat enthielt. Durch Behandeln mit Alkohol konnten daraus unveränderter Harnstoff sowie geringe Mengen anderer orgánischer zum Teil schwefelfreier Substanzen gewonnen werden, die jedoch nicht analysenrein erhalten werden konnten, weshalb von ihrer näheren Untersuchung Abstand genommen wurde. Die von Kutschig beschriebene und analysierte Verbindung war darin gewiß nicht in irgendwie erheblicher Menge vorhanden, da einerseits bei ihrer großen Kristallisationsfähigkeit ihre Abscheidung hätte gelingen müssen, anderseits aber auch der hohe Schwefelgehalt die Substanz auch als Beimengung verraten hätte; diesbezügliche Versuche wurden angestellt, blieben aber erfolglos. Es wurde nun zur näheren Untersuchung der vorhin erwähnten, zuerst ausgefallenen Kristalle I geschritten. Die Kristalle waren in heißem Wasser sehr leicht, in kaltem erheblich schwerer löslich, so daß sich Wasser sehr gut zumUmkristallisieren eignet. Einen eigentlichen Schmelzpunkt besaß die Substanz nicht, sondern sie zersetzt sich je nach der Schnelligkeit des Erhitzens bei verschiedenen Temperaturen unter Aufschäumen. Der Zersetzungspunkt liegt bei beiläufig $230^{\circ}$. Die Verbindung enthielt geringe Mengen von Kristallwasser, die bei $100^{\circ}$ unter teilweiser Zersetzung der Substanz entweichen.

Die Analyse lieferte folgende Zahlen:

I. $0.622 g$ lufttrockene Substanz verloren bei $104^{\circ} 0.0367 \mathrm{~g} \mathrm{H}_{2} \mathrm{O}$.

II. $0.5752 \mathrm{~g}$ lufttrockene Substanz gaben nach der Oxydation mit Bromwasser $0.8960 \mathrm{~g} \mathrm{BaSO}_{4}$.

Anmerkung. Sämtliche Schwefelbestimmungen wurden in der Weise durchgeführt, daß die Substanz mit Bromwasser oxydiert wurde. Ich habe mich durch eigene Versuche überzeugt, daß die in dieser Arbeit beschriebenen Verbindungen hiebei allen Schwefel in Form von Schwefelsäure liefern, denn die Zahlen stimmten völlig mit den nach anderen Methoden (Oxydation mit $\mathrm{HNO}_{3}+\mathrm{KClO}_{3}$, Asbóth's Methode mit $\mathrm{Na}_{2} \mathrm{O}_{2}$ ) erhaltenen überein.

III. $0 \cdot 4323 \mathrm{~g}$ bei $110^{\circ}$ getrocknete Substanz gaben $0.6282 \mathrm{~g} \mathrm{BaSO}_{4}$ und $0 \cdot 1592 \mathrm{~g} \mathrm{Mg}_{2} \mathrm{P}_{2} \mathrm{O}_{7}$.

IV. $0.2900 \mathrm{~g}$ bei $110^{\circ}$ getrocknete Substanz gaben $80.9 \mathrm{~cm}^{3}$ feuchten $\mathrm{N}$ bei $722 m m$ und $14^{\circ} \mathrm{C}$.

V. $0.3124 \mathrm{~g}$ bei $110^{\circ}$ getrocknete Substanz gaben $0.1033 \mathrm{~g} \mathrm{H}_{2} \mathrm{O}$ und $0 \cdot 1665 \mathrm{~g} \mathrm{CO}_{2}$, 
In 100 Teilen:

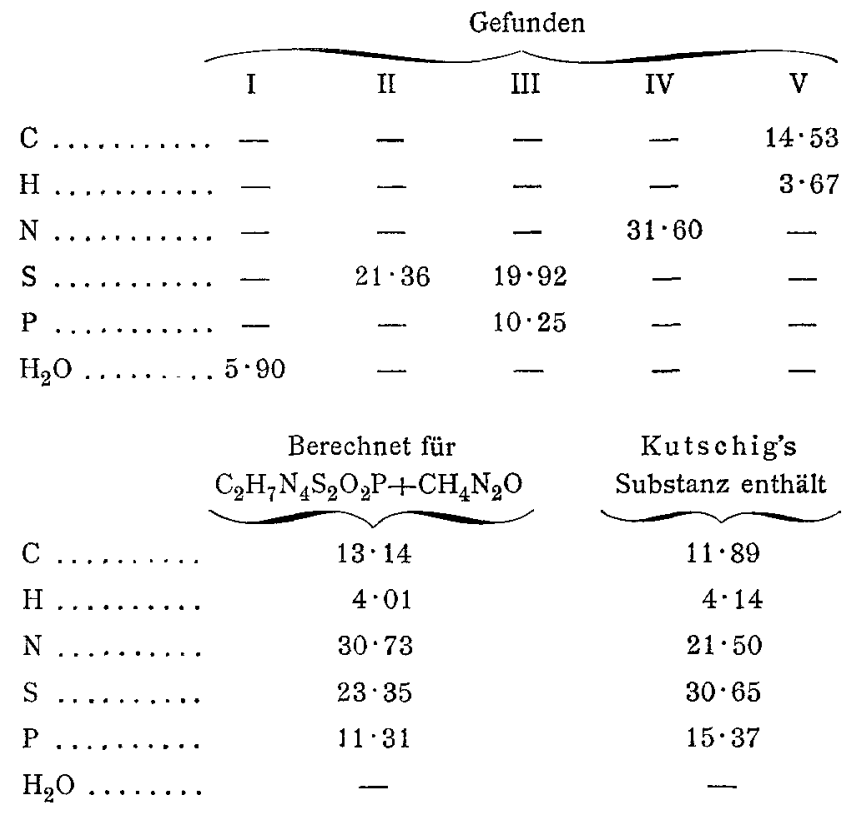

Die Verbindung weicht also in ihrer Zusammensetzung wesentlich von der von Kutschig gefundenen ab. Wie der hohe Stickstoff- und Kohlenstoffgehalt beweist, muß der Harnstoffkomplex noch ziemlich erhalten geblieben sein. Die berechneten Werte beziehen sich auf eine Verbindung von Harnstoff mit dem Ammoniumsalz der später zu beschreibenden, bei energischer Einwirkung von Phosphorpentasulfid auf Harnstoff entstehenden Thiobiuretphosphorsäure. Eine solche Verbindung ist immerhin denkbar, da die genannte Säure dreibasisch ist, doch glaube ich annehmen zu müssen, daß eine einfache Salzbildung mit dem Harnstoff hier gewiß nicht vorliegt. Einerseits ist die Übereinstimmung der berechneten Werte mit den gefundenen keine gute, andrerseits konnte ich aber auch aus dem thiobiuretphosphorsauren Ammonium und Harnstoff kein derartiges Salz erhalten. Es muß demnach wohl irgend eine innigere Bindung mit dem Harnstoff hier vorliegen. Ich wollte durch die Berechnung der Werte auch nur zeigen, daß die Reaktion hier ihr Ende noch nicht erreicht hat, sondern ein dem Harnstoff noch näher stehendes Zwischenprodukt vorliegt. 


\section{Zweiter Versuch.}

$50 \mathrm{~g}$ Harnstoff wurden mit $100 \mathrm{~g}$ gepulvertem Phosphorpentasulfid innigst verrieben und hierauf in einem Kolben im Wasserbade solange erhitzt, bis der Kolbeninhalt in eine zähflüssige Masse verwandelt war, die bei weiterem Erhitzen sich nicht mehr wesentlich aufblähte. Der Kolben war dabei mit einem Tuche bedeckt und sein Inhalt wurde öfter mit einem Glasstabe durchgerührt. Beim Übergießen mit Wasser trat wieder starke Schwefelwasserstoffentwicklung ein; es wurde nun solange auf dem Wasserbad erwärmt, bis die Gasentwicklung aufhörte, dann filtriert und das Filtrat eingedampft. Beim Erkalten scheiden sich größere Mengen von Kristallen aus, die je nach der Konzentration der ursprünglichen Lösung noch wechselnde Mengen von Ammoniumphosphat enthalten. Einmaliges Umkristallisieren aus heißem Wasser genügt, um letzteres abzuscheiden und die Substanz rein zu gewinnen. Ausbeute 8 bis $9 g$. Ich erwähne gleich an dieser Stelle, daß es durchaus nicht nötig ist, eine so große Menge von Phosphorpentasulfid anzuwenden; ich habe bei Anwendung gleicher Gewichtsmengen von Harnstoff und Phosphorpentasulfid dieselbe Ausbeute erhalten und nur deshalb anfangs mit dem Überschuß gearbeitet, um dem Einwande zu begegnen, daß ich Kutschig's Vorschrift nicht befolgt hätte. Es ist also auch gewiß unrichtig, wenn Kutschig angibt, daß in seinem Reaktionsprodukt kein unverändertes Phosphorpentasulfid mehr vorhanden war. Aus der Mutterlauge der oben beschriebenen Substanz konnte bei weiterem Eindampfen wesentlich nur Ammonitumphosphat gewonnen werden, so sehr ich mich auch bemühte, dabei die von Kutschig beschriebenen Kristalle zu erhalten.

Dagegen gab die erste Ausscheidung, die also schwerer löslich ist als das Ammoniumphosphat, beim langsamen Verdunsten der wässerigen Lösung Kristalle, die durch ihr schönes Aussehen mich sofort auf die Vermutung brachten, daß hier der Kutschig'sche Körper vorliege. Ich habe" mich deshalb an Herrn Prof. Gol dschmiedt mit der Bitte gewandt, die Messung der Kristalle beziehungsweise den Vergleich mit den seinerzeit 
von Hern Kohn gemessenen zu vermitteln. Die von Herrn Dr. Stuchlik im Institute von Herrn Prof. Pelikan angestellten Versuche ergaben nun tatsächlich die Identität der beiden Substanzen.

Herr Dr. Stuchlik teilte mir hierüber folgendes mit:

"Die Kristalle des thiobiuretphosphorsauren Ammoniums zeigten den angegebenen Habitus: monoklin, tafelförmig nach der Basis oder der Längsfläche; beobachtet wurde das Auftreten der gleichen Flächen mit folgenden gemessenen Winkelwerten (Durchschnittswerte, teilweise mangelhafte Reflexe):

$\begin{array}{rll}c(001): b & (010)=90^{\circ} & \text { Kohn's Werte: } \\ : m(110)=80^{\circ} 1^{\prime} & 80^{\circ} 23^{\prime} \\ : r^{\prime}(\overline{2} 01)=65^{\circ} 43^{\prime} & 65^{\circ} 34^{\prime} 34^{\prime \prime} \\ : p^{\prime}(\overline{1} 11)=48^{\circ} 23^{\prime} & 48^{\circ} 19^{\prime} \\ : o(11)=38^{\circ} 58^{\prime} & 39^{\circ} 7^{\prime} 36^{\prime \prime} \\ m(110): m^{\prime \prime}(1 \overline{1} 0)=73^{\circ} 33^{\prime} & 73^{\circ} 37^{\prime}\end{array}$

Berechnet wurde der Winkel $\beta=77^{\circ} 41^{\prime} \quad 77^{\circ} 57^{\prime}$.

Es kann demnach keinem Zweifel unterliegen, daß meine Substanz mit der Kutschig's identisch war; warum sie sich bei Kutschig's Versuchen nach dem phosphorsauren Ammonium ausgeschieden hat, ist mir ganz unverständlich, da ich dies trotz zahlreicher Versuche nie beobachten konnte. Die Substanz zeigte im allgemeinen auch die von Kutschig angegebenen Eigenschaften in Bezug auf Aussehen (meine Substanz wurde zwar fast immer sofort farblos erhalten) und Löslichkeit.

Beim Erhitzen in der Kapillare tritt bei $252^{\circ}$ stürmische Zersetzung ein. Einen Geruch nach Schwefelwasserstoff konnte ich bei der trockenen Verbindung nicht beobachten, hingegen tritt der Geruch beim Lösen in Wasser sofort auf. Beim Erhitzen 'mit Kalilauge entwickelt sich Ammoniak; es liegt demnach ein Ammoniumsalz vor. Mit Magnesiamischung entsteht kein Niederschlag, das Phosphorsäureion wird also aus der Verbindung nicht abgespalten.

Die Analyse führte zu folgenden Zahlen:

I. $0.6906 \mathrm{~g}$ bei $100^{\circ}$ getrocknete Substanz (kein Gewichtsverlust) gaben $1 \cdot 4870 \mathrm{~g} \mathrm{BaSO}_{4}$ und $0.3535 \mathrm{~g} \mathrm{Mg}_{2} \mathrm{P}_{2} \mathrm{O}_{7}$. 
II. $0.2588 \mathrm{~g}$ trockene Substanz gaben $60.6 \mathrm{~cm}^{3}$ feuchten $\mathrm{N}$ bei $17^{\circ}$ und $740 \mathrm{~mm}$.

III. $0.3012 \mathrm{~g}$ trockene Substanz gaben $0.0922 \mathrm{~g} \mathrm{H}_{2} \mathrm{O}$ und $0.1258 \mathrm{~g} \mathrm{CO}_{2}$. (Die Substanz wurde im Schiffchen mit geschmolzenem $\mathrm{K}_{2} \mathrm{Cr}_{2} \mathrm{O}_{7}$ bestreut.)

In 100 Teilen:

\begin{tabular}{|c|c|c|c|}
\hline & \multicolumn{2}{|c|}{ Gefunden } & \multirow{2}{*}{$\begin{array}{l}\text { Berechnet für } \\
\mathrm{C}_{2} \mathrm{H}_{7} \mathrm{PO}_{2} \mathrm{~S}_{2} \mathrm{~N}_{4}\end{array}$} \\
\hline & Hemmelmayr & Kutschig & \\
\hline $\mathrm{C} \ldots \ldots \ldots$ & $11 \cdot 38$ & $11 \cdot 89$ & $11 \cdot 21$ \\
\hline$H \ldots \ldots \ldots$ & $3 \cdot 40$ & $4 \cdot 14$ & $3 \cdot 27$ \\
\hline$N \ldots \ldots \ldots$ & $26 \cdot 46$ & $21 \cdot 50$ & $26 \cdot 17$ \\
\hline$s \ldots \ldots$ & $29 \cdot 52$ & $30 \cdot 65$ & $29 \cdot 90$ \\
\hline$P \ldots \ldots \ldots$ & $14 \cdot 25$ & $15 \cdot 37$ & $14 \cdot 48$ \\
\hline
\end{tabular}

Meine Werte weichen besonders beim Stickstoff ziemlich beträchtlich von denen Kutschig's ab; ich habe deshalb Substanzen, die von verschiedenen Darstellungen herrührten, wiederholt analysiert, erhielt jedoch stets Werte, die mit meinen vorhin angegebenen sehr gut übereinstimmten $(\mathrm{S}=30 \cdot 22$, $29 \cdot 91,30 \cdot 35,30 \cdot 28 ; \mathrm{P}=14 \cdot 31,14 \cdot 05 ; \mathrm{C}=11 \cdot 01 ; \mathrm{H}=3 \cdot 27)$. Ich bin deshalb zu der Annahme gezwungen, daß Kutschig's Substanz nicht rein war, und zwar vermute ich, daß freie Säure beigemengt war, da nur dies den niedrigeren Stickstoffgehalt erklären kann.

Die gemessenen Kristalle werden übrigens ja auch bei Kutschig rein gewesen sein, es ist aber durch nichts bewiesen, daß nur solche analysiert wurden und daß nicht auch andere beigemengt waren.

Für meine Ansicht spricht auch der Umtand, daß Kutschig bei der Destillation der Substanz mit Natronlauge $8 \cdot 47 \%$ Ammoniak erhielt (für $1 \mathrm{NH}_{4}$ in der von mir zu Grunde gelegten Formel $\mathrm{C}_{2} \mathrm{H}_{7} \mathrm{PO}_{2} \mathrm{~S}_{2} \mathrm{~N}_{4}$ berechnet sich $7 \cdot 94 \% \mathrm{NH}_{3}$ ), was mit dem niedrigen Stickstoffgehalt nicht in Einklang zu bringen ist, da ja auch Kutschig überdies noch den Biuretkomplex mit drei Stickstoffatomen in seiner Verbindung auffand.

\section{Spaltung des Reaktionsproduktes mit verdünnter Salzsäure.}

Kutschig erhielt bei der Spaltung seiner Substanz durch heiße Salzsäure $(d=1 \cdot 1)$ Biuret und Phosphorsäure, wenn das Erhitzen bei Luftzutritt, Biuret und phosphorige Säure, wenn 
das Erhitzen im Kohlendioxydstrom geschah. Berücksichtigt man, daß phosphorige Säure bei ihrer Darstellung in salzsaurer Lösung in offenen Gefäßen eingedampft wird, ohne daß sie dabei in erheblicher Menge in Phosphorsäure übergeht, so ist die Bildung der phosphorigen Säure bei obiger Zersetzung sehr wenig wahrscheinlich. Ich habe deshalb den Versuch wiederholt und habe dabei folgendes Resultat erhalten:

$5 \mathrm{~g}$ Substanz wurden mit $80 \mathrm{~cm}^{3}$ Salzsäure $(d=1 \cdot 1)$ eine Stunde am Rückflußkühler im Kohlendioxydstrom gekocht. Die Flüssigkeit, die anfangs viel Schwefelwasserstoff abgab, riecht nach dieser Zeit nicht mehr nach diesem Gase und gibt wohl die Reaktionen der Phosphorsäure, nicht aber die der phosphorigen Säure. Es entsteht also auch bei Luftabschluß nur Phosphorsäure.

Die Flüssigkeit wurde nun auf dem Wasserbade eingedampft und dann ins Vakuum gestellt. Zuerst fällt Salmiak aus, von dem abgesaugt wurde. Die Mutterlauge gibt nach längerem Stehen wieder Kristalle, die nach dem Umkristallisieren aus Wasser feine Nadeln bilden, die bei $193^{\circ}$ schmelzen (Biuret Schmelzpunkt $=190^{\circ}$ ) und mit Kupfersulfat und Natronlauge die Biuretreaktion gaben. Ein anderes Spaltungsprodukt konnte nicht aufgefunden werden.

Im Hinblick auf den Zerfall der Substanz in Biuret und Phosphorsäure und auf ihren Schwefelgehalt schlage ich für die freie Säure den Namen Thiobiuretphosphorsäure vor; das Reaktionsprodukt zwischen Harnstoff und Phosphorpentasulfid ist dementsprechend als thiobiuretphosphorsaures Ammonium zu bezeichnen.

\section{Salze der Thiobiuretphosphorsäure.}

Das bei der Reaktion zwischen Harnstoff und Phosphorpentasulfid entstehende Ammoniumsalz reagiert noch sauer. Ich habe deshalb versucht, ein neutrales Ammoniumsalz herzustellen.

$\mathrm{Zu}$ diesem $\mathrm{Zwecke}$ wurden $8 \mathrm{~g}$ des sauren Ammoniumsalzes in Wasser gelöst, $60 \mathrm{~cm}^{3}$ Ammoniak $(d=0.930)$ und soviel Alkohol zugesetzt, daß sich die Flüssigkeit gerade trübte. Es scheiden sich glänzende prismatische Kristalle aus, die 
selbst in vollkommen trockenem Zustande stark nach Ammoniak riechen.

Nach eintägigem Liegen an der Luft verloren $0.4873 \mathrm{~g}$ bei $104^{\circ} 0.0338 g$ an Gewicht, entsprechend $7.45 \%$. Nach achttägigem Liegen an der Luft war der Geruch nach Ammoniak verschwunden und es fand jetzt auch beim Erhitzen auf $104^{\circ}$ kein Gewichtsverlust mehr statt. Eine Stickstoffbestimmung ergab folgende Werte:

$0.2720 \mathrm{~g}$ bei $104^{\circ}$ getrocknete Substanz gaben $64.4 \mathrm{~cm}^{3}$ feuchten $\mathrm{N}$ bei $16^{\circ}$ und $735 \mathrm{~mm}$.

In 100 Teilen:

$$
N \ldots \ldots \ldots \underbrace{\text { Gefunden }}_{26 \cdot 68} \quad \underbrace{\begin{array}{c}
\text { Berechnet für } \\
\mathrm{C}_{2} \mathrm{H}_{7} \mathrm{~N}_{4} \mathrm{PO}_{2} \mathrm{~S}_{2}
\end{array}}_{26 \cdot 17}
$$

Es ist also nur das primäre Ammoniumsalz der Thiobiuretphosphorsäure beständig, die anderen Ammoniumsalze verlieren schon bei längerem Liegen an der Luft einen Teil des Ammoniaks und gehen in das primäre über.

\section{Tertiäres Baryumsalz der Thiobiuretphosphorsäure.}

Die Herstellung dieses Salzes habe ich auf zwei Wegen versucht und bin dabei zum selben Resultat gelangt. Zuerst habe ich zu einer Lösung des Ammoniumsalzes solange filtriertes Barytwasser gesetzt, bis ein starker Überschuß an diesem vorhanden war. Anfangs löste sich der entstandene Niederschlag beim Schütteln wieder auf und erst größere Mengen von Barytwasser bewirkten einen bleibenden Niederschlag. Es ist also das zuerst entstehende saure Salz im Wasser löslich, womit auch der Umstand in Übereinstimmung steht, daß das Ammoniumsalz durch Baryumchlorid nicht gefällt wird. Der Niederschlag wurde nun abgesaugt, mit Wasser gewaschen und getrocknet; selbstverständlich wurden alle diese Operationen unter möglichstem Ausschluß von Kohlendioxyd durchgeführt. Trotzdem aber wurde der Baryumgehalt etwas zu hoch gefunden.

Ich habe deshalb einen bequemeren Weg eingeschlagen und eine warme Lösung des Ammoniumsalzes mit einer 
Mischung von Ammoniak und Baryumchlorid (am besten nimmt man etwas mehr als die berechneten Mengen) versetzt. Beim Erkalten fäilt dann das tertiäre Baryumsalz in glänzenden, blättrigen Kristallen aus, die durch Absaugen und Waschen mit kaltem Wasser analysenrein gewonnen werden. Die Kristalle reagieren stark alkalisch. Beim Kochen mit Kaliumhydroxyd entweicht kein Ammoniak, Salzsäure löst leicht auf. Auf Zusatz von starker Salpetersäure findet stürmische Oxydation statt, ebenso wird auch durch Bromwasser die salzsaure Lösung sofort unter Abscheidung von Baryumsulfat getrübt. Das Baryumsalz enthält Kristallwasser, von dem ein Teil schon beim Stehen im Exsikkator entweicht.

Bei der Analyse wurde so vorgegangen, daß die drei Elemente Baryum, Schwefel und Phosphor in derselben Substanzmenge bestimmt wurden. Zuerst wurde das Salz mit Wasser übergossen, eine Spur Salzsäure (so daß sich das Salz gerade löst) und dann sofort eine größere Menge Bromwasser zugesetzt. Nachdem auf dem Wasserbade das Brom weggedampft worden war, wurde vom Baryumsulfat abfiltriert, das Filtrat mit Baryumchlorid gefällt und durch ein neues Filter filtriert. Im Filtrat wurde nach Entfernen des Baryumüberschusses, wie üblich, die Phosphorsäure bestimmt. Die zuerst gefundene Menge Baryumsulfat diente zur Berechnung des Baryums, die Gesamtmenge an Baryumsulfat zur Berechnung des Schwefelgehaltes.

Es wurden folgende Werte erhalten:

I. $0.5965 \mathrm{~g}$ lufttrockenes Saiz verloren bei $125^{\circ} 0.0935 \mathrm{~g}$ Wasser.

II. $0.3891 \mathrm{~g}$ luftrockenes Salz gaben zuerst $0.2716 \mathrm{~g}$, dann nach Zusatz von Baryumchlorid noch $0.1029 \mathrm{~g}$, im ganzen also $0.3745 \mathrm{~g} \mathrm{BaSO}_{4}$ und $0.0788 \mathrm{~g} \mathrm{Mg}_{2} \mathrm{P}_{2} \mathrm{O}_{7}$.

III. $0.2678 \mathrm{~g}$ lufttrockenes Salz (mit Barytwasser bereitet) gaben $0.1925 \mathrm{~g}$ $\mathrm{BaSO}_{4}$.

IV. $0.3748 \mathrm{~g}$ lufttrockenes Sal7, (mit Barytwasser bereitet) gaben $0.3594 \mathrm{~g}$ $\mathrm{BaSO}_{4}$ (mit $\mathrm{HNO}_{3}$ oxydiert!) und $0.0885 \mathrm{~g} \mathrm{Mg}_{2} \mathrm{P}_{2} \mathrm{O}_{7}$.

V. $0.4454 \mathrm{~g}$ bei $125^{\circ}$ getrocknetes Baryumsalz gaben zuerst $0.3707 \mathrm{~g}$, dann nach Zusatz von $\mathrm{BaCl}_{2}$ noch $0.1310 \mathrm{~g}$, im ganzen also $0.5017 \mathrm{~g}$ $\mathrm{BaSO}_{4}$ und $0.1218 \mathrm{~g} \mathrm{Mg}_{2} \mathrm{P}_{2} \mathrm{O}_{7}$.

VI. $0.5422 \mathrm{~g}$ bei $125^{\circ}$ getrocknetes Salz gaben $49.8 \mathrm{~cm}^{3}$ feuchten $\mathrm{N}$ bei $21^{\circ}$ und $740 \mathrm{~mm}$. 
VII. $0 \cdot 4285 g$ lufttrockenes Salz (im Schiffchen mit $\mathrm{K}_{2} \mathrm{Cr}_{2} \mathrm{O}_{7}$ gemischt, gaben $0.0817 \mathrm{~g} \mathrm{CO}_{2}$. (Die Wasserstoffbestimmung ging durch Zufall verloren.)

In 100 Teilen:

\begin{tabular}{|c|c|c|c|c|c|c|c|}
\hline & \multicolumn{7}{|c|}{ Gefunden } \\
\hline & I & II & III & IV & V & $\mathrm{VI}$ & VII \\
\hline $\mathrm{C} \ldots \ldots \ldots$ & - & - & - & 一 & - & - & $5 \cdot 19$ \\
\hline $\mathrm{N} \ldots \ldots$ & - & -- & - & - & - & $10 \cdot 17$ & - \\
\hline$s \ldots \ldots \ldots$ & - & $13 \cdot 20$ & - & $13 \cdot 16$ & $15 \cdot 58$ & - & 一 \\
\hline$P \ldots \ldots, \ldots$ & - & $5 \cdot 63$ & - & $6 \cdot 59$ & $7 \cdot 61$ & - & - \\
\hline $\mathrm{Ba} \ldots \ldots \ldots$ & - & $41 \cdot[0$ & $42 \cdot 26$ & - & $49 \cdot 00$ & - & - \\
\hline $\mathrm{H}_{2} \mathrm{O} \ldots \ldots \ldots$ & $5 \cdot 68$ & - & - & - & - & - & - \\
\hline
\end{tabular}

\begin{tabular}{lcc}
\multicolumn{3}{c}{ Berechnet für } \\
\multicolumn{2}{c}{$\mathrm{C}_{4} \mathrm{H}_{6} \mathrm{~S}_{4} \mathrm{~N}_{6} \mathrm{P}_{2} \mathrm{O}_{6} \mathrm{Ba}_{3}+81 / 2 \mathrm{H}_{2} \mathrm{O}$} & $\mathrm{C}_{4} \mathrm{H}_{6} \mathrm{~S}_{4} \mathrm{~N}_{6} \mathrm{P}_{2} \mathrm{O}_{6} \mathrm{Ba}$ \\
$\mathrm{C} \ldots \ldots \ldots$ & $5 \cdot 04$ & - \\
$\mathrm{S} \ldots \ldots \ldots$ & - & $10 \cdot 04$ \\
$\mathrm{P} \ldots \ldots \ldots$ & $12 \cdot 94$ & $15 \cdot 31$ \\
$\mathrm{Ba} \ldots \ldots \ldots$ & $6 \cdot 27$ & $7 \cdot 42$ \\
$\mathrm{H}_{2} \mathrm{O} \ldots \ldots \ldots$ & 41.65 & $49 \cdot 28$
\end{tabular}

Außer den angeführten Analysen habe ich noch eine große Anzahl anderer von Baryumsalzen verschiedener Darstellung gemacht, habe aber dabei stets die gleichen Resultate erlangt (im kristallwasserhaltigen Salze: $\mathrm{S}=13 \cdot 10,12 \cdot 89 ; \mathrm{P}=6 \cdot 61$; $\mathrm{Ba}=41 \cdot 45,41 \cdot 37,41 \cdot 33$; im wasserfreien Salze: $\mathrm{S}=15 \cdot 53$, $\mathrm{Ba}=48 \cdot 98$ ). Ich glaube daher berechtigt zu sein, das Salz als rein $z u$ bezeichnen.

Es ist demnach die Thiobiuretphosphorsäure tatsächlich eine dreibasische Säure. Aus der Analyse des Baryumsalzes geht aber auch hervor, daß die Bildung der tertiären Salze unter gleichzeitiger Aufnahme eines Moleküls Wasser erfolgt. Abgesehen davon, daß sonst der Baryumgehalt wesentlich höher sein müßte (für $\mathrm{C}_{4} \mathrm{H}_{2} \mathrm{P}_{2} \mathrm{~S}_{4} \mathrm{~N}_{6} \mathrm{O}_{4} \mathrm{Ba}_{2}$ berechnet sich $51.51 \%$ $\mathrm{Ba}$ statt der gefundenen Menge von $49.00 \%$ ), wäre bei dem geringen Wasserstoff- und Sauerstoffgehalt der freien Säure es schwer verständlich, daß sie dreibasisch sein könnte. Ein höherer Gehalt an Sauerstoff und Wasserstoff in der freien Säure beziehungsweise dem primären Ammoniumsalz würde 
aber Unterschiede besonders im Schwefelgehalt bedingen, die weit die gewöhnlichen Analysenfehler übersteigen.

Anschließend möchte ich erwähnen, daß das Silbersalz, das Kutschig durch Fällung seiner Substanz mit Silberacetat erhielt, bei der Analyse Zahlen lieferte, die gut für das primäre Silbersalz der Thiobiuretphosphorsäure stimmen. Mit Kutschig's Formel war die Übereinstimmung besonders im Silbergehalt schlechter, was er auf Zersetzung des Salzes zurückführte. Ich gebe die Zahlen hiemit kurz an:

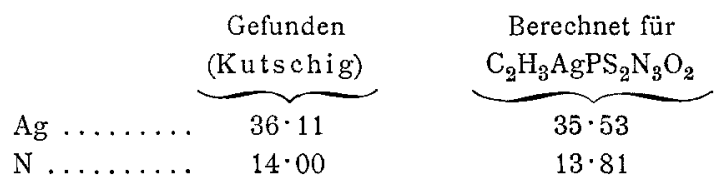

Kutschig erhielt noch ein zweites Silbersalz mit Hilfe einer ammoniakalischen Silberlösung; Analysen teilte er nicht mit, da das Salz sehr lichtempfindlich ist. Ich habe es deshalb auch vorgezogen, die Basizität mit Hilfe des Baryumsalzes zu bestimmen.

\section{Darstellung der freien Thiobiuretphosphorsäure.}

Behufs Darstellung der freien Säure wurde das tertiäre Baryumsalz mit der berechneten Menge von Halbnormalschwefelsäure übergossen, nach längerem Stehen und zeitweiligem Umschütteln vom Baryumsulfat abfiltriert und das Filtrat im Vakuum verdunsten gelassen.

Die Kristallisation begann erst, als nur noch eine ganz geringe Menge Flüssigkeit vorhanden war; es schieden sich dann farblose Kristallkrusten aus, die gut abgesaugt und mit ganz wenig Wasser gewaschen wurden. Die Mutterlauge roch stark nach Schwefelwasserstoff und auch die Kristalle zeigten einen schwachen Geruch. Die Verbindung ist in Wasser sehr leicht löslich, reagiert stark sauer und gibt keine Reaktion auf Phosphorsäure; sie ist auch in Alkohol leicht löslich. Beim Erhitzen in der Kapillare tritt bei $78^{\circ}$ Zersetzung ein, die immer lebhafter wird, und bei $86^{\circ}$ scheinbar beendet ist. Im Exsikkator verlieren die Kristalle ihre Durchsichtigkeit und verwandeln 
sich in eine weiße Masse; offenbar enthält die kristallisierte Substanz demnach Kristallwasser. Zur Analyse wurde die lufttrockene Verbindung verwendet, da es nicht kontrollierbar war, ob im Exsikkator nur Wasser abgegeben wurde oder ob eine weitergehende Zersetzung stattfand; um so weniger konnte an ein Trocknen bei höherer Temperatur gedacht werden.

Die Analyse gab folgende Zahlen;

I. $0 \cdot 2626 g$ lufttrockene Substanz gaben $0.5352 g \mathrm{BaSO}_{4}$.

II. $0.6099 \mathrm{~g}$ lufttrockene Substanz (von einer anderen Darstellung herrührend) gaben $1 \cdot 2432 \mathrm{~g} \mathrm{Ba} \mathrm{SO}_{4}$.

In 100 Teilen:

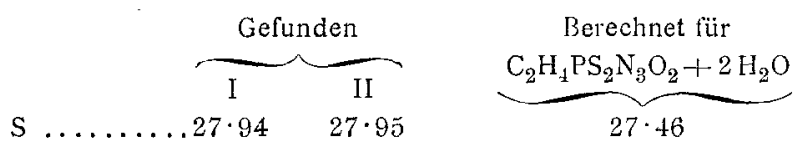

Es lag demnach die freie Thiobiuretphosphorsäure mit 2 Molekülen Kristallwasser vor.

Um zu entscheiden, ob bei der Überführung in das Baryumsalz und der darauffolgenden Zersetzung des Salzes mit Schwefelsäure nicht doch eine tiefergehende Umwandlung stattfindet, wurde die Lösung der freien Säure mit Ammoniak versetzt und dann im Vakuum über Schwefelsäure verdunsten gelassen. Es empfiehlt sich nicht, die ammoniakalische Flüssigkeit einzudampfen, da hiebei teilweise Zersetzung eintritt. Die zuerst ausfallenden Kristalle sind zwar rein, die Mutterlauge enthält aber Phosphorsäure, was beim Verdunsten im Vakuum nicht der Fall ist. Die Analyse der auch im Aussehen dem thiobiuretphosphorsauren Ammonium gleichenden Kristalle führte zu folgenden Werten:

$0.3829 \mathrm{~g}$ Substanz gaben $0.8477 \mathrm{~g} \mathrm{BaSO}_{4}$ und $0.1933 \mathrm{~g} \mathrm{Mg}_{2} \mathrm{P}_{2} \mathrm{O}_{7}$.

In 100 Teilen:

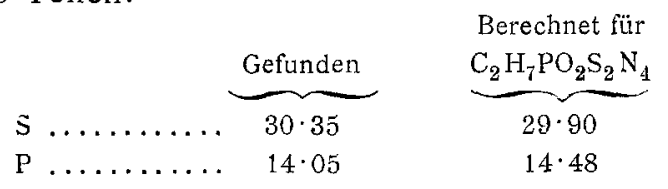

Es war also wieder thiobiuretphosphorsaures Ammonium entstanden. 


\section{Einwirkung von Phosphorpentasulfid auf Thioharnstoff.}

$30 \mathrm{~g}$ Thioharnstoff und $20 \mathrm{~g}$ Phosphorpentasulfid wurden innig verrieben und in einem Glaskolben im Paraffinbade auf $130^{\circ}$ erhitzt. Schon bei $120^{\circ}$ beginnt sich die Mischung aufzublähen; nach längerem Erhitzen stellt sie eine zähflüssige blasige Masse vor. Diese wurde mit Wasser übergossen, einige Zeit auf dem Wasserbad erwärmt und hierauf filtriert. Das Filtrat liefert beim Eindampfen zunächst große weiße Kristalle, die nach dem Umkristallisieren aus heißem Wasser bei $174^{\circ}$ schmelzen; sie enthalten keinen Phosphor.

$0.5339 \mathrm{~g}$ gaben nach der Oxydation mit Bromwasser $1.6297 \mathrm{~g} \mathrm{BaSO}$.

In 100 Teilen:

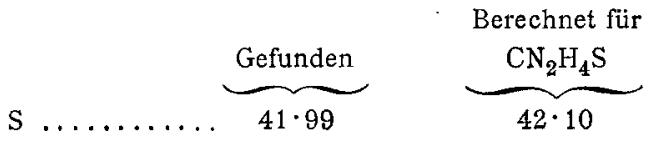

Die Substanz war demnach Thioharnstoff.

Die Mutterlauge von der ersten Kristallausscheidung liefert bei weiterem Eindampfen eine Flüssigkeit, die sich beim Erkalten in zwei Schichten trennt, eine untere ölige (I) und eine darüberstehende wässerige (II). Das Öl erstarrt langsam zu Kristallen, die unscharf bei $118^{\circ}$ schmelzen. Nach dem Umkristallisieren erhält man neben etwas Ammoniumphosphat eine sehr geringe Menge organischer, schwefelhältiger Substanz, die aber nicht näher untersucht wurde, da zu wenig davon vorhanden war. Übrigens hat diese Substanz jedenfalls für die Reaktion nur geringe Bedeutung, da ihre Menge in keinem Verhältnis zum aufgewendeten Thioharnstoff steht.

Der wässerige Anteil (II) sowohl als auch das Filtrat von (I) reagierten stark sauer; sie wurden mit Ammoniak versetzt, was bedeutende Erwärmung und Ausscheidung von Ammoniumphosphat zur Folge hatte; auch färbte sich die Flüssigkeit hiebei grün. Das ausgeschiedene Ammoniumphosphat erwies sich als fast schwefelfrei; eine größere Menge eines schwefelhaltigen Reaktionsproduktes konnte also auch hier nicht enthalten sein. 
Die Mutterlauge vom Ammoniumphosphat gab bei weiterem Eindampfen neuerdings Kristalle, die durch Behandeln mit Alkohol einen unlöslichen Rückstand, bestehend aus Ammoniumphosphat, gaben. Die alkoholische Lösung liefert beim Eindampfen einen mit Kristallen durchsetzten Sirup. Die Kristalle wurden auf Ton gestrichen und aus Wasser umkristallisiert; sie zeigten den Schmelzpunkt $173^{\circ}$, enthielten viel Schwefel, aber keinen Phosphor und waren zweifellos Thioharnstoff. Da ihre Mutterlauge starke Rhodanreaktion gab, war der Syrup vermutlich der Hauptsache nach Rhodanammonium, das sich durch Umlagern des Thioharnstoffes beim Erhitzen gebildet hatte.

Wurde der Thioharnstoff mit dem Phosphorpentasulfid noch höher erhitzt $\left(160^{\circ}\right)$, so bestand das Reaktionsprodukt (nach dem Zersetzen mit Wasser) vorwiegend aus Rhodanammonium und Ammoniumphosphat. Überdies wurde noch eine verschwindend kleine Menge einer bei $186^{\circ}$ schmelzenden Verbindung erhalten, die große gelbliche Kristalle bildete, aber für eine genaue Untersuchung nicht ausreichte.

Den Herren Prof. Dr. Goldschmiedt und Dr. Pelikan sowie Herrn Dr. Stuchlik danke ich auch an dieser Stelle herzlich für die Vermittlung der Kristalmessung, ebenso Herrn Prof. Dr. O. Hecht für die Übersendung seines Methyldithiobiurets. 